\title{
EXECUTIVE FUNCTIONING AND DELINQUENT BEHAVIOR IN CHINESE JUVENILE DELINQUENT WITH COMORBID DEVELOPMENTAL READING DISABILITY AND ATTENTION-DEFICIT/HYPERACTIVITY DISORDER
}

K.Y. Poon

Department of Psychology, The University of Hong Kong, Hong Kong, Hong

Kong

BACKGROUND: Many studies reported high prevalence of attention deficit hyperactivity disorder (ADHD) and reading disorder (RD) among offenders. Very few have examined the group with comorbid ADHD+RD and its underlying cognitive profile. The present study was to compare the severity of delinquent behavior and executive function (EF) in a group of juvenile delinquent with ADHD, RD, and comorbid ADHD+ RD. METHOD: Subjects were adolescent boys with RD and/or ADHD recruited from juvenile institutions. Assessment batteries on executive functions (EF) were administered, and subscale on delinquent behavior of Teachers Report Form (TRF) was completed. One-way analyses of variance (ANOVA) with post-hoc analysis of group differences was employed comparing three groups of juvenile delinquents (29 ADHD, 24 RD, 35 ADHD+ RD) and 29 controls on their delinquent behavior and EF. RESULTS: ADHD+RD had a significantly high score in delinquent behavior comparing to pure groups. Comparing with controls, both pure ADHD and RD were associated with poor visual search, inhibition and planning, whereas pure RD showed additional poor processing speed. The comorbid ADHD+RD group shared all deficits with pure groups, and in addition, it exhibited a unique deficit in interference control and severed impairment in processing speed.

CONCLUSION: The relationship between delinquency and executive functions shown for the ADHD+RD groups pointed to a distinct executive dysfunction of the comorbid condition. EF should be considered in the remediation protocol. http://dx.doi.org/10.1016/j.neurenf.2012.04.120 